\title{
Rethinking the Treatment of Pathologic Scars
}

\author{
Rahman Barry*1, Peter Ray ${ }^{1}$ and Hadiyah-Nicole Green*2 \\ ${ }^{1}$ Department of Surgery, Marshall University, Huntington, West Virginia
}

${ }^{2}$ Birmingham VA Medical Center, Birmingham, Alabama; Morehouse School of Medicine, Atlanta, Georgia; Ora Lee Smith Cancer Research Foundation, Atlanta, Georgia

Received: 䟧 January 07, 2019; Published: 非January 16, 2019

*Corresponding author: Rahman Barry, Department of Surgery, Marshall University, Huntington, West Virginia

Hadiyah-Nicole Green, Birmingham VA Medical Center, Birmingham, Alabama; Morehouse School of Medicine, Atlanta, Georgia; Ora Lee Smith Cancer Research Foundation, Atlanta, Georgia

\section{Opinion}

Over 100 million people develop a pathologic scar every year worldwide [1] Attempts to treat scars in the United States alone account for almost \$15 Billion of expenditures with a large percentage of patients left with scars so symptomatic and impactful on daily life that they continue to seek treatment [2]. The Scar Free Foundation's 2015 mandate to deliver scar free healing within a generation is a testament to the magnitude of this problem. Traditional theories about the causes of pathologic scar include fibroblast overgrowth, collagen overproduction, dysfunctional local immune response, presence of poorly encapsulated loose particles, endocrine irregularities, over or underproduction of cytokine mediators and presence of unique inflammatory cells. Recent understanding of the pathophysiology of scar is that it results from failure to progress through the normal phases of healing, from the inflammatory phase to the proliferative phase to the remodeling phase. It remains unknown how the dominant cells of each phase "hand off" to the dominant cells of the next phase and allow the wound to progress without becoming locked into a pro-fibrotic state that cannot resolve itself.

In clinical practice, patients arrive with scars of highly variable etiology, time course and symptomatology which makes it impossible to establish rigid research protocols. Current scar treatment protocols include steroid injections, silicone pressure therapy, immunosuppressive agent injections, cryotherapy, surgery, radiation and laser as well as other less common methods. Some non-surgical methods of treatment include compression garments, massage, pulsed light, steroids, exercise and fat injections. The decision of which therapy to apply against a given scar is often the result of the most senior authority's informed opinion or rigid adherence to an established protocol which accepts variable outcomes. Internationally recognized experts agree that current therapies are "not enough" [3]. Many clinical studies can show improvement on a given scar scale, but there is a persistently low adoption rate of any given protocol into clinical practice. The design of randomized controlled trials to match and compare therapies has been complicated by the wide variation of causal factors, demographics and lapse in time since the original injury. The impact of this level of imprecision and complexity is frustrating to both patients and practitioners alike as they try to match the right scar with the right treatment to get consistent and effective results. What works in one patient scenario may not work in the next. As a result, there exists a bewildering array of research-based and direct-to-consumer products available for use in the management of both pathologic and non-pathologic scars. This level of variation and complexity needs a more accurate and precise underlying organizational approach to unify research efforts and guide treatment choices. We see a need to develop a method of matching treatment to the specific scar of a specific patient at a specific time point in the scar's maturation course. A modern "patient specific" technology that is a more simplified, scar specific approach to treatment would bridge the use of personalized medicine and targeted therapies for patients with pathologic scars as well as nonpathologic scars.

Since the smallest functional, biological unit in a scar is the individual cell, we advance the opinion that the field should focus on the persistent cellular response within the scar by selectively deleting the maladaptive cells with a patient-specific targeted therapy. By focusing on the responding cells, rather than on the 
external stimuli, there is a potential to implement targeted therapies to address "the greatest unmet need" of pathologic scarring. This concept simply challenges the traditional ways of thinking about pathologic scarring to open new avenues of research and apply novel technologies to an age-old problem. We aim to encourage clinicians to move beyond the "phases of wound healing" construct and use patient-specific and scar-specific functional histologic data combined with a cell-targeting technology. This approach is certain to lead us towards a deeper understanding of the wound microenvironment and open new possibilities for more effective treatments of routine scarring, pathologic scarring, or rare genetic scar-related conditions without the comorbidities of radiation or steroid use. We envision a world where untargeted therapies are a thing of the past.

\section{ISSN: 2574-1241}

DOI: 10.26717/BJSTR.2019.13.002362

Rahman Barry, Hadiyah-Nicole Green. Biomed J Sci \& Tech Res

CC (i) This work is licensed under Creative

Submission Link: https://biomedres.us/submit-manuscript.php

\section{Acknowledgment}

The project described was supported by Award Number I01BX007080 from the Biomedical Laboratory Research \& Development Service of the VA Office of Research and Development.

\section{References}

1. Sund B (2000) New developments in wound care. PJB pp. 1-255.

2. Fearmonti RM, Bond JE, Erdmann D, Levin LS, Pizzo SV, et al. (2011) The modified patient and observer scar assessment scale: A novel approach to defining pathologic and nonpathologic scarring. Plast Reconstr Surg 127(1): 242-247.

3. Finnerty CC, Jeschke MG, Branski LK, Barret JP, Dziewulski P, et al. (2016) Hypertrophic scarring: The greatest unmet challenge after burn injury. Lancet 388(10052): 1427-1436.

$\begin{array}{ll}\text { BIOMEDICAL } & \text { Assets of Publishing with us } \\ \text { RESEARCHES } & \text { - Global archiving of articles } \\ \text { - Immediate, unrestricted online access }\end{array}$

